\title{
Computational Intelligence for Transportation: Driving Safety and Assistance
}

\author{
Wuhong Wang \\ Department of Transportation Engineering, Beijing Institute of Technology, Beijing 100081, PR China \\ wangwuhong@bit.edu.cn \\ Klaus Bengler \\ Institute of Ergonomics, Technische Universität München, Munich, D-85747, Germany
}

The continuously increasing features of sensor technology and data processing make intelligent transportation system (ITS) widely applied in ground transportation and various vehicles systems in many countries. Since ITS is addressing heavily serious environmental and safety concerns through an integrative approach that encompasses the entire transportation paradigm, the advance driver assistance systems (ADAS) has been considered as a greatly important part of the solution to safety problems in traffic system. For current and further research, the analyzing, testing, modeling and simulating of driver behavior have been conducting for the development of ADAS, and the innovative technologies will collectively improve fuel efficiency and safety of various vehicle, all which will be used to reduce emissions, manufacturing costs, traffic accidents and traffic congestion.

In the traffic operation the ADAS has been playing an active role in decreasing the number of traffic accidents. The ADAS might cover a full range of systems varying from the systems of providing mobility information to the systems of controlling vehicle operation. Some typical examples are collision warning systems, lane departure warning systems, ACC (Adaptive Cruise Control system), vision enhancement and pedestrian detection. Nowadays the development of computing and tracking technology enables deployment of new ADAS and makes the driving task more comfortable and safe. Further improvements will be made in the integration of driver assistance systems and traffic safety control. As we all expected a safe, reliable, economic driver assistance system will materialize an accident-free traffic in the foreseeable future. As a result, the international symposium on green intelligent transportation system and sino-german workshop on traffic safety through driver assistance has been organized to review the state of knowledge in the area and define future directions for research aimed at preventing or mitigating the consequences of driving errors in traffic operation.

The symposium aimed at presenting and showing the new achievement of technologies and theories in traffic safety and advance driver assistance systems; the current traffic problems and solutions; the trend of the development of driving intelligence and vehicle safety control. The symposium was intended to promote a better communication for all the participants, and lay a basis for interdisciplinary efforts of controlling traffic accidents. The symposium has offered the opportunity to stimulate new research directions and exchange ideas with many of the experts. The output from the collective efforts of all participants is captured in this special issue of International Journal of Computational Intelligence Systems. The special issue on computational intelligence for transportation includes the 15 final revised and extended papers, which show the current development in the field of green intelligent transportation systems and the state of the art of traffic safety through driver assistance. This special issue is just for appreciations of authors' cooperative participants of the symposium. 